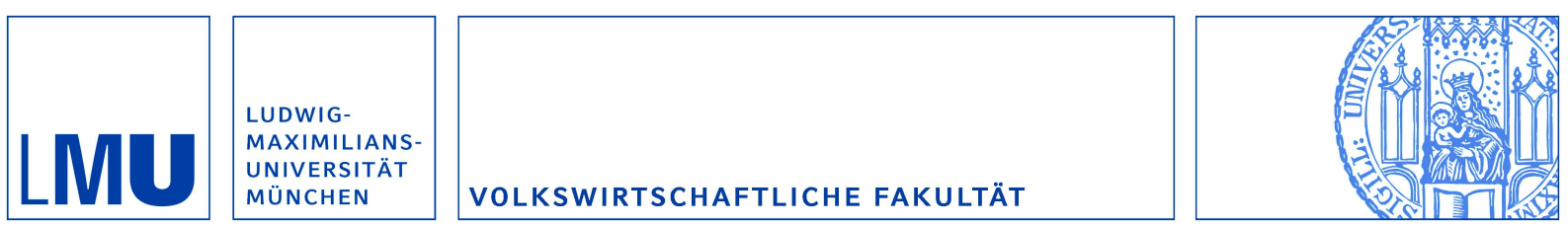

Kocher, Martin G.; Lenz, Marc V. und Sutter, Matthias:

Psychological pressure in competitive environments: Evidence from a randomized natural experiment: Comment

Munich Discussion Paper No. 2010-18

Department of Economics

University of Munich

Volkswirtschaftliche Fakultät

Ludwig-Maximilians-Universität München

Online at https://doi.org/10.5282/ubm/epub. 11445 


\title{
Psychological pressure in competitive environments: Evidence from a randomized natural experiment: Comment ${ }^{*}$
}

\author{
Martin G. Kocher \\ University of Munich and University of Innsbruck \\ Marc V. Lenz \\ University of Cologne \\ Matthias Sutter \\ University of Innsbruck, University of Gothenburg, and IZA Bonn
}

\begin{abstract}
Apesteguia and Palacios-Huerta (forthcoming) report for a sample of 129 shootouts from various seasons in ten different competitions that teams kicking first in soccer penalty shootouts win significantly more often than teams kicking second. Collecting data for the entire history of six major soccer competitions we cannot replicate their result. Teams kicking first win only $53.4 \%$ of 262 shootouts in our data, which is not significantly different from random. Our findings have two implications: (1) Apesteguia and Palacios-Huerta's results are not generally robust. (2) Using specific subsamples without a coherent criterion for data selection might lead to non-representative findings.
\end{abstract}

JEL-classification: C93

Keywords: $\quad$ Tournament, first-mover advantage, psychological pressure, field experiment, soccer, penalty shootouts

This version: 22 March 2010

* We are particularly indebted to Gary Charness, David Cooper, Uri Gneezy, Lorenz Goette, Steven Levitt, Bradley Ruffle and John Wooders for excellent comments.

\# Corresponding author. Address: Department of Public Finance, University of Innsbruck, Universitaetsstrasse 15, A-6020 Innsbruck, Austria. e-mail: matthias.sutter@uibk.ac.at 
A recent paper by Jose Apesteguia and Ignacio Palacios-Huerta (forthcoming; in the following APH) reports a systematic and significant first-mover advantage in soccer penalty shootouts. As APH convincingly argue, soccer penalty shootouts are an important example for the consequences of a puzzling psychological bias in sequential tournaments. More specifically, they say that their "results provide support for a source of psychological pressure that has a detrimental effect on performance, and that is different from others such as high stakes, social pressure or peer pressure previously documented in the literature" (p. 15f. of the accepted manuscript).

APH have collected data on penalty shootouts from ten different national and international competitions (World Cup, Continental Cups, National Cups). Based on their collection of 129 shootouts, APH show that the first-kicking teams win in 78 cases $(60.5 \%)$, and lose only in 51 cases ( $p=0.022$; two-sided Binomial test), despite the a priori probability of both teams winning with a $50 \%$ chance. APH ascribe this very strong and significant effect of sequential moves in a tournatment to psychological pressure on the second-kicking teams, because they typically face an intermediate score in the shootout that is to their disadvantage when kicking.

The striking findings of APH are at odds with the results of an earlier publication of ours. In Martin G. Kocher, Marc V. Lenz and Matthias Sutter (2008) we examined the determinants of scoring in penalty shootouts in the German DFB-Pokal (German Soccer Cup Competition), but our focus in that paper was not on a possible first-mover advantage. Nevertheless, for a preliminary data set of 95 easily accessible penalty shootouts in the seasons from 1986/1987 to $2006 / 2007$ we found that the first-kicking teams won in only $48.4 \%$ of cases. This was not significantly different from the expected $50 \%$, and hence different from the more recent results of APH.

Intrigued by this inconsistency and interested in providing a robustness test of APH's results, we considerably extended our data set by collecting data from six major competitions and the penalty shootouts therein from 1970 onwards. First, we collected data for the entire history of the German Soccer Cup Competition, which ranks among Europe's most important soccer competitions. Then we gathered data on the three most important European-level club competitions (and their predecessors), i.e., the European Champions League, the UEFA Cup, and the European Cup Winners' Cup. Finally, we took shoutouts from the two most important soccer events in the world, the European Championship and the World Cup, both of which are competitions for national teams.

Our approach regarding data collection is different from the one used by APH. We collected data for the entire history of six major competitions. APH consider more 
competitions (ten), but they select subsamples of each competition that belong to different time periods (see their Table 1). While APH take into account (almost) all shootouts in competitions of national teams (i.e., World Cup, European Championship, American Cup, African Nations Cup, Gold Cup), the time periods that they chose for the various club competitions do not reveal a systematic pattern or rule for inclusion. For the Champions League and the UEFA Cup, they consider the years 2000-2003 plus all finals. For the Spanish Cup they use data from 1999 to 2003, and all prior finals. For the German Cups (German Soccer Cup Competition and German Supercup) they take the years 2001-2003 as well as all prior finals. Finally, for the English Cups their paper lacks information on the years being taken into account.

For the six competitions that we consider, we have data for 262 shootouts that took place from 1970 to June 2003. In restricting our dataset to shootouts before summer 2003 we follow the convincing argument of APH that only until then shootout data can be used for an unbiased examination of a potential first-mover advantage (see section 1 below for details), Of the 262 shootouts that we consider, the first-kicking teams won a total of 140. The relative frequency of $53.4 \%$ is by far not significantly different from the a priori expected probability of 50\% ( $p=0.29$; two-sided Binomial test). Hence, the results of APH are not generally robust, and the seemingly minor detail of which time period to consider makes a big difference for finding a first-mover advantage or not.

The rest of the comment is organized as follows. In section 1 we describe our data set, the sources we used and the rules that determine which team kicks first in a penalty shootout. Section 2 presents our results, and section 3 concludes.

\section{Rules and Data}

Penalty shootouts were introduced by the world governing body of soccer, the Fédération Internationale de Football Association (FIFA) in 1970 to determine the winner in knock-out tournament games in which there was a tie between two teams after 90 minutes of regular time and 30 minutes of overtime. Before 1970, the winner in tied games in a knock-out tournament was determined by the draw of a lot.

The basic rules for a penalty shootout are as follows (for details see the official "Rules of the Game" at www.fifa.com): First, each team selects five players (out of the players on the pitch in the $120^{\text {th }}$ minute). Second, teams kick in alternating order. Third, the shootout is terminated as soon as the number of penalties converted by one team cannot be matched by the other team. If - after both teams have taken five kicks - both have scored the same 
number of goals, teams continue kicking in the same alternating order until one team has scored a goal more than the other from the same number of kicks (i.e., in a one-on-one competition). Each penalty kick during the shootout must be taken by a different player, and all eligible players must take a kick before any player can take a second kick. In principle, this sequence can go on infinitely. However, the longest shootout in our data set includes 26 kicks, i.e., 13 for each team. ${ }^{1}$

From 1970 until June 2003 the first-kicking team was the team that won the referee's toss of a coin before the shootout. Such a rule constitutes an explicit randomization to determine the beginning team. ${ }^{2}$ In July 2003 , the FIFA changed the rules slightly by giving the winner of the referee's toss of a coin the option of choosing whether to kick first or second. This choice option may give rise to endogeneity problems, and therefore we only use data for 1970 to June 2003 (i.e., the end of the season 2002/2003).

Our data of the shootouts in the German Soccer Cup Competition have been retrieved by resorting to the print and online editions of Kicker Sportmagazin, the most important magazine for German football (see www.kicker.de), the online data archive www.fussballdaten.de, and in a few cases by calling clubs' headquarters. In total, we were able to collect the relevant data for 105 out of a total of 115 shootouts in the entire period from 1970 to June 2003. We are missing details on the order of kicking in ten shootouts in the 1970s and 1980s because, due to professional printers being on strike, the Kicker Sportmagazin was forced to distribute some incomplete issues, and it was impossible to find other sources for the order of kicking in the ten cases.

The data for the European Champions League (and its predecessor, the European Champion Clubs' Cup), the UEFA Cup (and its predecessor, the European Fairs Cup), and the European Cup Winners' Cup (which ceased to be played out in 1999) were taken from Ionescu (2003, 2004a, 2004b, 2004c, 2004d, 2004e). In total, there were 138 shootouts in

\footnotetext{
${ }^{1}$ A soccer penalty shootout belongs to a class of games that Mark Walker, John Wooders, and Rabah Amir (2009) call binary Markov games. For a theoretical account of such games, see their paper. Penalty shootouts are a good example for studying mixed strategies in games (see Pierre-Andre Chiappori, Steven Levitt and Timothy Groseclose, 2002). Consequently, soccer players - because of their penalty shooting experience - have been used to examine mixed strategy play of professionals in the laboratory (see Palacios-Huerta and Oscar Volij, 2008; Levitt, John List and David Reiley, forthcoming; Wooders, forthcoming).

${ }^{2}$ In the terminology of Glenn Harrison and List (2004) this rule constitutes a truly randomized natural experiment.
} 
these three club competitions on the European level from 1970 to June 2003, and we were able to find data on the kicking order for 132 shootouts.

For the World Cup and the European Championship we collected the necessary data for all 25 shootouts that ever took place in these competitions from 1970 to 2002. Sources were the Kicker Sportmagazin, www.fifa.com, and www.fussballdaten.de.

\section{Results}

Table 1 presents the data for all six competitions. The first column shows the number of shootouts for which we have data. The second column indicates the relative frequency with which the first-kicking teams won the shootout in the relevant competition. The third column reports the $p$-value from a two-sided Binomial test that examines whether the observed relative frequency of first-kicking teams winning the shootout is significantly different from $50 \%$.

Table 1 and Figure 1 about here

For none of the six competitions we find a significant first-mover advantage. The relative frequency with which the first-kicking teams win the shootout varies from $33 \%$ in the European Championship to $63 \%$ in the Champions League. If we pool all data (see final row of Table 1), the Binomial test gives a $p$-value of 0.29 for our sample of 262 shootouts.

If we exclude the data from the German Soccer Cup Competition - and therefore concentrate exclusively on the international competitions - the $p$-value of the Binomial test is $0.26(N=157)$, showing clearly that our null-result is not driven by the German data. It is noteworthy that World Cups and European Championships are also included in the data set of $\mathrm{APH}$, but they do not report separate statistics for them. Hence, the main result established in APH is not driven by these high-stakes competitions, either. If psychological pressure is at all strong in penalty shootout situations, it must be strongest in these competitions, as they attract the most public attention and media coverage, and they are of the utmost importance for the standing and career prospect of every kicker on the pitch. However, out of the 25 shootouts in the World Cup and the European Championship before 2003, only ten were won by the firstkicking team. ${ }^{3}$

\footnotetext{
${ }^{3}$ For a paper addressing the influence of incentives on experimental laboratory behavior see Dan Ariely, Uri Gneezy, George Loewenstein, and Nina Mazar (2009).
} 
Figure 1 presents the relative frequency of the first-kicking teams winning and the number of shootouts in all seasons from 1970/1971 (denoted 1970 in Figure 1) to 2002/2003 (denoted 2002). ${ }^{4}$ According to a Binomial test, the relative frequency of first-kicking teams to win the shootout is not significantly different from 50\% in any single year. From Figure 1 it becomes clear that the relative frequency with which first-kicking teams win the shootout is larger than $50 \%$ in 12 seasons, and smaller than $50 \%$ in another 12 seasons. $^{5}$

\section{Conclusion}

Penalty shootouts from 1970 until June 2003 constitute a truly randomized experiment. Taking a look at the influence of the order of kicking on the probability of winning allows studying a possible first-mover advantage that might be due to psychological pressure on the team that kicks second. Considering the whole history of six major competitions (the World Cup, the European Championship, the European Champions League, the UEFA-Cup, the European Cup Winners' Cup, and the German Soccer Cup Competition), we did not find significant evidence of a first-mover advantage. Considering all 262 shootouts from 1970 to June 2003 the first-kicking teams won in $53.4 \%$ of cases, which is not significantly different from the expected a priori probability of 50\%. Likewise, none of the six competitions separately yields a result that is statistically different from $50 \%$, nor do we find a single year in which first-kicking teams win significantly more often than second-kicking teams. Hence, our findings are in marked contrast to the results presented by Apesteguia and PalaciosHuerta (forthcoming), who report that first-kicking teams win significantly more often than $a$ priori expected, i.e., in $60.5 \%$ of 129 shootouts in ten different national and international competitions, where the time period considered in their paper is different across competitions.

\footnotetext{
${ }^{4}$ The World Cups and European Championships take place every four years, typically from June to early July. These competitions are then regarded as the end of a season. Therefore, the World Cup 2002 is part of the season 2001/2002, showing up in the bar "2001" in Figure 1. The number of shootouts is generally smaller in the 1970s and 1980s due to a change in rules in the German Soccer Cup Competition. Prior to 1991 the rules stipulated to play a second match (in the other team's stadium) if a match ended with a draw after 120 minutes. Only if there was again a draw after 120 minutes in the second leg, a penalty shootout had to determine the winner. From 1991 on, the possibility of a second match was abolished and each cup match had to be decided in a shootout if it was tied after 120 minutes.

${ }^{5}$ A randomized binomial test (see Wooders, 2008, for details how to calculate it) cannot reject the null hypothesis that the first-kicking team's winning probability is $50 \%$, either. This implies that the observed winning frequencies in the seasons from 1970/1971 to 2002/2003 are conistent with a random chance of the firstkicking team winning the shootout. We would like to thank John Wooders for drawing our attention to the test.
} 
To conclude, we think that there are two main implications from this comment: First, the findings and conclusions offered by Apesteguia and Palacios-Huerta (forthcoming) on the first-mover advantage in sequential tournaments do not appear to be robust and, therefore, are less general than it seems at first sight. Second, taking specific subsamples without a clear and coherent criterion on which time periods and competitions to include could lead to nonrepresentative results. 


\section{References}

Apesteguia, Jose, and Ignacio Palacios-Huerta. Forthcoming. "Psychological Pressure in Competitive Environments: Evidence From a Randomized Natural Experiment.” American Economic Review.

Ariely, Dan, Uri Gneezy, George Loewenstein, and Nina Mazar. 2009. "Large Stakes and Big Mistakes." Review of Economic Studies 76(2): 451-470.

Chiappori, Pierre-Andre, Steven Levitt, and Timothy Groseclose. 2002. Testing MixedStrategy Equilibria When Players are Heterogeneous: The Case of Penalty Kicks in Soccer. American Economic Review 92(4): 1138-1151.

Harrison, Glenn, and John A. List. 2004. "Field Experiments." Journal of Economic Literature XLII(4): 1013-1059.

Ionescu, Romeo. 2003. The Complete Results \& Line-Ups of the European Fairs Cup 19551971. Cleethorpes: Soccer Books Limited.

Ionescu, Romeo. 2004a. The Complete Results \& Line-Ups of the European Champion Clubs' Cup 1955-1991 - The Knockout Years. Cleethorpes: Soccer Books Limited.

Ionescu, Romeo. 2004b. The Complete Results \& Line-Ups of the European Champions League 1991-2004. Cleethorpes: Soccer Books Limited.

Ionescu, Romeo. 2004c. The Complete Results \& Line-Ups of the European Cup Winners' Cup 1960-1999. Cleethorpes: Soccer Books Limited.

Ionescu, Romeo. 2004d. The Complete Results \& Line-Ups of the UEFA Cup 1991-2004. Cleethorpes: Soccer Books Limited.

Ionescu, Romeo. 2004e. The Complete Results \& Line-Ups of the UEFA Cup 1971-1991. Cleethorpes: Soccer Books Limited.

Kocher, Martin G., Marc V. Lenz, and Matthias Sutter. 2008. "Performance Under Pressure - The Case of Penalty Shootouts in Football.” In: Patric Andersson, Peter Ayton, and Carsten Schmidt (eds.). Myths and Facts about Football: The Economics and Psychology of the World's Greatest Sport. Cambridge: Cambridge Scholars Publishing: 61-72.

Levitt, Steven, John List, and David Reiley. Forthcoming. "What Happens in the Field Stays in the Field: Professionals Do Not Play Minimax in Laboratory Experiments." Econometrica.

Palacios-Huerta, Ignacio, and Oscar Volij. 2008. "Experientia Docet: Professionals Play Minimax in Laboratory Experiments.” Econometrica 76(1): 71-115. 
Walker, Marc, John Wooders, and Rabah Amir. 2009. "Equilibrium Play in Matches: Binary Markov Games.” University of Arizona, Working Paper.

Wooders, John. Forthcoming. "Does Experience Teach? Professionals and Minimax Play in the Lab." Econometrica.

Wooders, John. 2008. "Does Experience Teach? Professionals and Minimax Play in the Lab.” University of Arizona, Department of Economics. Working Paper. 


\section{Table and Figure}

Table 1: Shootout data for six major competitions and all seasons from 1970/1971 to 2002/2003

\begin{tabular}{lccc}
\hline \hline & $\begin{array}{c}\text { Number of } \\
\text { shootouts }\end{array}$ & $\begin{array}{c}\text { First-kicking team wins } \\
\text { (relative frequency) }\end{array}$ & $\begin{array}{c}\text { p-value (two-sided } \\
\text { Binomial test) }\end{array}$ \\
\cline { 2 - 4 } [1] German Soccer Cup Competition & 105 & 0.514 & 0.85 \\
[2] European Champions League* & 27 & 0.630 & 0.25 \\
[3] European Cup Winners' Cup ${ }^{\#}$ & 32 & 0.625 & 0.22 \\
[4] UEFA-Cup & 73 & 0.534 & 0.64 \\
[5] European Championship & 9 & 0.333 & 0.51 \\
[6] World Cup & 16 & 0.438 & 0.80 \\
Sum [1]- [6] & 262 & 0.534 & 0.29 \\
\hline \hline
\end{tabular}

Notes:

${ }^{\dagger}$ There are 10 more shootouts in the German Soccer Cup Competition for which we lack data on the order of kicks.

* The data for this competition include its predecessor, the European Champion Clubs' Cup, which was played out from 1955-1991. There is one more shootout with missing data on the kicking order.

" This competition ceased in 1999. There are two more shootouts for which we miss data on the kicking order.

* The data for this competition include its predecessor, the European Fairs Cup, which was played out from 1955-1971. There are three more shootouts for which we miss data on the kicking order. 
Figure 1: Relative frequency of first-kicking teams winning a shootout - data from all competitions combined (number of shootouts in parenthesis)

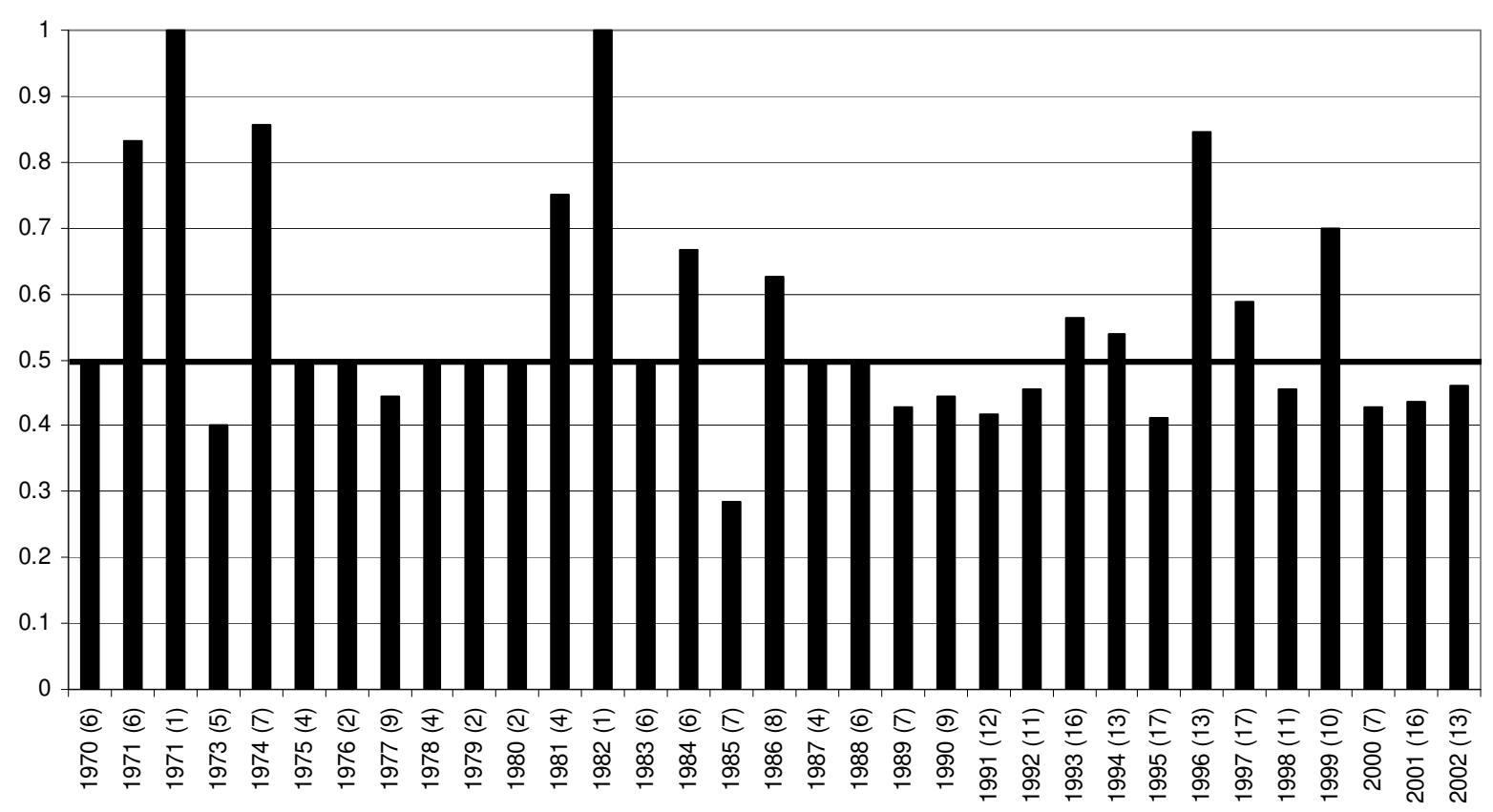

